
Revue canadienne de chimie

\title{
From optimized monovalent ligands to size-controlled dendrimers: an efficient strategy towards high-activity DC- SIGN antagonists
}

\begin{tabular}{|r|l|}
\hline Journal: & Canadian Journal of Chemistry \\
\hline Manuscript ID & cjc-2017-0138.R1 \\
\hline Manuscript Type: & Mini Review \\
\hline Date Submitted by the Author: & 11 -Apr-2017 \\
\hline $\begin{array}{r}\text { Complete List of Authors: } \\
\text { Ordanini, Stefania; Universita degli Studi di Milano, Dipartimento di } \\
\text { Chimica; Politecnico di Milano Dipartimento di Chimica Materiali e } \\
\text { Ingegneria Chimica Giulio Natta } \\
\text { Goti, Giulio; Universita degli Studi di Milano, Dipartimento di Chimica } \\
\text { Bernardi, Anna; Universita degli Studi di Milano, Dipartimento di Chimica }\end{array}$ \\
\hline $\begin{array}{r}\text { Is the invited manuscript for } \\
\text { Issue? Please select:: }\end{array}$ & Dendimers \\
\hline Keyword: & DC-SIGN, glycomimetics, glycodendrimers, HIV, multivalency \\
\hline \multicolumn{2}{|c}{} \\
\hline
\end{tabular}




\section{From optimized monovalent ligands to size-controlled dendrimers: an}

2 efficient strategy towards high-activity DC-SIGN antagonists

3 Stefania Ordanini ${ }^{+}$, Giulio Goti, Anna Bernardi*

4 Department of Chemistry, Università degli Studi di Milano, Via C. Golgi 19, 20133, Milan, $5 \quad$ Italy

6 + Current address: Department of Chemistry, Materials and Chemical Engineering "G.

$7 \quad$ Natta”, Politecnico di Milano, via Mancinelli 7, 20131, Milan, Italy

$8 \quad$ * E-mail: anna.bernardi@unimi.it; Tel: +39 02 50314092; Fax: +39 0250314072

9 


\section{Abstract}

11 This short review describes our work on the development of dendrimeric antagonists of DC12 SIGN, a Dendritic Cells (DCs) receptor recognizing highly mannosylated structures and 13 primarily involved in the recognition of viruses, such as HIV. The structure of pseudo-dimannoside and pseudo-tri-mannoside compounds was first finely modified to obtain DCSIGN ligands more stable and selective than mannose. Their DC-SIGN affinity differences were amplified once presented on multivalent dendrimer-like scaffolds, including poly-alkyne terminated and phenylene-ethynylene rod-like ones. Libraries of mannosylated dendrimers were synthesized, improving their stability and maximizing their monodispersity. The effect of the dendrimers valency, structure and size on DC-SIGN affinity and antiviral potency was investigated. Both the valency and the topology of the architectures were revealed as key parameters for activity optimization, together with the intrinsic affinity of the monovalent ligand. The stability, rigidity and length of the scaffolds were also tuned. The design of geometrically adapted scaffolds afforded one of the most potent inhibitors of DC-SIGNmediated HIV infections to date. This monodispersed, not cytotoxic and highly active compound was also tested with DCs; its internalization into endolysosomal compartments and its ability to induce the overexpression of signaling molecules makes it a good precursor to produce pathogen-entry inhibitors with immunomodulant properties.

Key words (3-5): DC-SIGN, glycomimetics, glycodendrimers, HIV, multivalency. 


\section{Introduction}

Human immunodeficiency virus (HIV) is still a huge health problem of the $21^{\text {st }}$ century, causing the death of over 1 million people per year from AIDS-related illnesses ${ }^{1}$. Waiting for an effective HIV vaccine ${ }^{2}$, the search for HIV entry-inhibitors represents a promising strategy to prevent HIV infection. HIV mainly targets Dendritic Cells (DCs), monocyte/macrophage lineage cells, and $\mathrm{CD}^{+}$T-lymphocytes ${ }^{3}$. Both receptor-mediated and non-specific electrostatic interactions drive the contact between the virus and the host cells. The discovery of artificial molecules able to efficiently antagonize one or a combination of these interactions goes in the direction of producing powerful microbicides. Nano-sized compounds have shown promising potential as effective inhibitors of viral infections ${ }^{4}$. Several classes of dendrimers have been designed and used to block different HIV-host cells interactions, targeting either cellular receptors or the viral envelope; their application as HIV entry-inhibiting microbicides has been recently reviewed ${ }^{5}$.

In this short review, we will describe the research that has been carried out in our group for the past decade, focusing on the discovery of microbicides that operate by antagonizing the dendritic cell receptor DC-SIGN. DC-SIGN (dendritic cell-specific ICAM-3 grabbing nonintegrin) is a trans-membrane lectin expressed at the surface of immature dendritic cells, particularly of dermal and mucosal tissue, and involved in the early stages of HIV infection ${ }^{6}$. DC-SIGN recognizes also other pathogens, including Ebola or Dengue viruses, promoting viral transmission and dissemination ${ }^{7}$. This lectin is a tetramer presenting four carbohydrate recognition domains (CRDs) that bind to highly mannosylated and fucosylated structures in a calcium-dependent manner. As for many other lectins, the affinity of DC-SIGN for its glycan ligands is only weak ${ }^{8}$ and strong binding is obtained, both in Nature and by synthetic antagonists, using multivalency. DC-SIGN antagonists have been produced functionalizing various multivalent platforms, including dendrimers, with mannose and oligomannoses ${ }^{9,10}$. In 
parallel, our strategy consisted first of the design and the synthesis of unnatural monovalent glycoconjugate mimetics, more complex and possibly more active and selective than mannose itself. Secondly, the most promising candidates were used for the synthesis of polyvalent constructs, mostly based on dendrimers or dendron scaffolds. The effect of the dendrimers valency, structure and size on DC-SIGN affinity and antiviral potency has been investigated. For this endeavour we have used both real dendrimers/dendrons and dendrimer-inspired structures, i.e. molecules that lack a proper nucleus and repeated layers, but still are symmetrical and branched.

\section{Monovalent ligand optimization}

The high mannose glycan (Man) $)_{9}(\text { GlcNAc) })_{2}$ (Figure 1) is one of the main carbohydrate ligands recognized by DC-SIGN; it is found in multiple copies on the HIV envelope

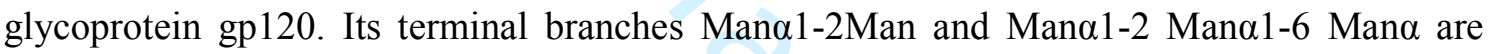
both involved in binding with the protein ${ }^{11}$, although recent studies indicate that the main interaction may occur through the oligosaccharide branched trimannose core ${ }^{12}$.

We have shown that pseudo-di-mannoside $\mathbf{1}^{13,14}$ and pseudo-tri-mannoside $\mathbf{2}^{15,16}$ (Figure 2), where one of the mannose residues has been replaced by an appropriate conformationally constrained cyclohexane ring, perform as structural and functional mimics of the natural sugars, while being stabilized against enzymatic hydrolysis ${ }^{13}$.

Both ligands 1 and $\mathbf{2}$ bind to DC-SIGN in the carbohydrate recognition domain, as shown by the X-ray structure of their protein complexes ${ }^{17,18}$. The DC-SIGN affinity of these monovalent ligands was estimated by Surface Plasmon Resonance (SPR) competition experiments, whereby their ability to inhibit DC-SIGN binding to a highly mannosylated surface was measured. In these experiments, where both the lectin and the antagonists are free in solution, the pseudo-trisaccharide $2\left(\mathrm{IC}_{50} 125 \mu \mathrm{M}\right)$ was found to be ca. one order of magnitude more 
81 potent than the corresponding pseudo-disaccharide $\mathbf{1}^{16}$, which in turn has the same activity of 82 the natural sugar Man $\alpha 1-2 \mathrm{Man}\left(\mathrm{IC}_{50} \text { ca. } 1 \mathrm{mM}\right)^{17}$, but is significantly less cytotoxic in cellular 83 experiments ${ }^{14}$. Mimic 3 (Figure 2c), which is further functionalized on the aglycon, belongs to

84

\section{Dendrimer optimization}

Protein-carbohydrate interactions are relatively weak, with dissociation constants often lying in the low millimolar range. Nonetheless, high affinity can be achieved through multiple interactions. Therefore, in Nature lectin receptors present either multiple carbohydrate recognition domains or an oligomeric structure allowing multiple binding towards the target glycans, usually exposed in several copies as glycoconjugates forming polyglycosylated surfaces. Multivalent interactions affect lectin affinity and also modulate lectin selectivity. For these reasons, to compete with this kind of interactions and efficiently bind to natural proteins, artificial glycoconjugates should be multivalent ${ }^{20}$. The multivalent binding modes that can be exploited include chelation, i.e. the simultaneous binding of more than one protein binding site by a single multivalent system (Figure 3a), as well as other non-chelating effects, such as the statistical rebinding effect (Figure 3b), caused by enhancement of the local concentration of the active ligands, and the ability of the multivalent structures to cluster/aggregate receptors (Figure $3 \mathrm{c})^{21}$.

Notably, the density of sugar residues, the topology of presentation and the orientation have emerged as important parameters able to shape protein/carbohydrate interactions affinity and selectivity $^{22,23}$. In the design of polyglycosylated DC-SIGN antagonists we made use of dendrimeric core structures as valuable scaffolds for the multivalent presentation of ligands. 
These architectures enabled efficient generation of monodisperse constructs with control over valency and spatial arrangement. In particular, dendrimers were decorated with the optimized monovalent glycomimetic ligands $\mathbf{1 - 3}$, which have shown a significant affinity for DC-SIGN. In a first attempt, we investigated how valency and the nature of the monovalent ligands affect the affinity towards the receptor. We then tuned the stability, rigidity and length of the scaffolds, in order to produce ligands able to match the distance between two contiguous DCSIGN binding sites, therefore being able to undergo a plurality of multivalent mechanisms and enhance their bioactivity, while maintaining a moderate valency.

\section{Polyester (Boltorn-type) scaffolds}

The first class of multivalent glycocompounds was prepared in collaboration with the group of Javier Rojo (CSIC-Seville) using 2,2-bis(hydroxymethyl)propionic acid (bis MPA) derived polyester dendrons and dendrimers (Boltorn-type). Rather than using polydisperse commercial scaffolds, the polyesters were prepared in a monodisperse manner. Both the tetravalent dendron 4 and the 32-valent dendrimer 5 (Figure 4) were synthesized using bis MPA as building block, and introducing carboxylic acid residues at the dendrimer periphery by reaction with conveniently protected succinic acid moieties. Compounds $\mathbf{1}$ and $\mathbf{2}$, both tethered to an amino-terminated linker, were coupled to the dendrimers using HATU as the activating agent, in the presence of $N, N$-diisopropylethylamine. Final compounds 4.1, 4.2, 5.1 and 5.2 (Figure 4) were obtained in high yields; the tetravalent compounds were completely monodisperse, whilst both $\mathbf{5 . 1}$ and $\mathbf{5 . 2}$ were loaded with 30-32 copies of the ligands, on $\operatorname{average~}^{24}$.

These compounds were tested as DC-SIGN ligands in SPR competition assays. Results showed a dependency on multivalent presentation, since the full dendrimers $\mathbf{5 . 1}$ and $\mathbf{5 . 2}\left(\mathrm{IC}_{50}\right.$ $=1-2 \mu \mathrm{M})$ were two orders of magnitude more active than the corresponding tetravalent 
dendrons $4\left(\mathrm{IC}_{50}=120-220 \mu \mathrm{M}\right)$. Surprisingly, though, the nature of the monovalent ligand did not affect the affinity significantly, since constructs of similar valency showed similar $\mathrm{IC}_{50} \mathrm{~S}$, despite the one order of magnitude difference observed at the monovalent level between 1 and 2. The same phenomenon was observed in an Ebola infection model ${ }^{24}$ and by testing 4.1, 4.2, 5.1 and 5.2 in a cellular model of HIV infection ${ }^{18}$. These data prompted us to reexamine in more detail the interaction of 2 with DC-SIGN by a number of biophysical techniques, which allowed to conclude that the affinity measured for this molecule in our initial experiments was largely determined by a clustering mechanism in solution (Figure 3c). In other words, the two mannose units at both ends of $\mathbf{2}$ are able to interact simultaneously with two DC-SIGN tetramers by simultaneously binding to one CRD per tetramer ${ }^{18}$. This behavior leads to a high potency measured in all the experimental set ups where both the protein tetramer and $\mathbf{2}$ are in the same solution (e.g. in SPR competition experiments and in Isothermal Titration Calorimetry (ITC)). However, the clustering mechanism is lost when $\mathbf{2}$ is conjugated to a polyvalent scaffold, which prevents the reducing end mannose from reaching its target, and is also unlikely to be relevant in physiological conditions, where DC-SIGN is bound to the cellular membrane. As a result, further studies on pseudo-glycodendrimers targeted against DC-SIGN were mostly focused on the modified pseudo-dimannoside $\mathbf{3}$.

From a practical point of view, the tetravalent dendrons 4.1 and 4.2 represent a good compromise between affinity and synthetic effort. However, both the polyester dendron 4.1 and 4.2 suffer from chemical instability, since the unhindered succinyl ester moieties used to introduce carboxylic groups on the outer layer, are labile to nucleophiles, both under basic and mild acidic catalysis. For instance, $30 \%$ of 4.2 was hydrolyzed after $6 \mathrm{~h}$ in water solution at physiological $\mathrm{pH}$ (7.4, PBS buffer, NMR investigation), highlighting its limit for in vivo applications. MS analysis of the product confirmed that the hydrolysis occurred at the 
155

156

157

158

159

160

161

162

163

164

165

166

167

168

169

170

171

172

173

174

175

176

177

178

179

succinyl ester bond. Moreover, this instability prevents purification by reverse phase chromatography and hinders the scale up of the synthetic process.

In order to achieve stabilized versions of the hydrolytically unstable constructs 4 (Figure 4), the synthesis of the analogous tetravalent dendron $4 \mathbf{N}$ was undertaken (Scheme 1$)^{25}$. In $4 \mathbf{N}$ the labile succinyl ester bonds are replaced with more robust amide functionalities, that can be installed starting from the tetramine 6 (Scheme 1). A diglycolate linker 7, slightly longer and more hydrophilic than succinate, was selected in this design, thus preserving the water solubility of the molecule. Additionally, the length of the linker helped to minimize side reactions due to attack of the scaffold nitrogen atom over the activated carboxylic acids during the final functionalization of the dendron.

Condensation of the tetracid $\mathbf{4 N}$ with the amino-tethered 1a and $2 \mathrm{a}$ led to dendrons $\mathbf{4 N} .1$ and 4N.2 (Figure 5), which were found to be fully stable both to chromatographic conditions and in water solution at physiological $\mathrm{pH}$. These dendrons have also been tested as ligands for mouse Mannose Binding Lectin (MBL-C), a C-type lectin involved in the pathophysiology of brain ischemia/reperfusion injury ${ }^{26}$, and have shown activities similar to the lead 4.2.

Additionally, functionalization of the dendron focal point with an azido-terminated tether was adopted to allow the convergent synthesis of higher valency constructs. In particular, exploiting $\mathrm{Cu}(\mathrm{I})$ catalyzed azide-alkyne cycloaddition (CuAAC), 4N.1 was further connected to a pentaerythritol derived scaffold 8 (Scheme 2). The resulting 16-valent compound 4.4 N.1 was almost 2-orders of magnitude more active than its precursor in an MBL-C binding $\operatorname{assay}^{25}$.

\section{Polyalkyne terminated scaffolds}

In a different approach, to further improve the synthetic accessibility and chemical stability of the dendrimers, new polyalkyne terminated scaffolds, with a valency ranging from 2 to 6 , 
180

181

182

183

184

185

were synthesized ${ }^{27}$. They were then decorated with azide-bearing ligands $\mathbf{1 - 3}$, or with mannose as a control, through CuAAC. Nondegradable glycodendrons and glycodendrimers with a valency of 3, 4 or 6 (9-11, Figure 6$)$ were obtained, characterized by stable ether bonds and triazole connectors. In a convergent approach, the trivalent dendrons $\mathbf{9}$, bearing an azido group at the focal point, were used to build higher valency constructs $(6-, 9-, 12-$ and 18 valent 12.9, 13.9, 10.9 and 11.9) by combination with either the divalent, tetravalent or hexavalent scaffolds 12, 13, 10 and 11 (Figure 6). CuAAC allowed a full control over the targeted structure: all products were characterized by NMR spectroscopy and MALDI-MS analysis, and were stable for months in water solution.

Compounds affinity for DC-SIGN was evaluated through SPR inhibition assays and the relative inhibitory potency (R.I.P.) values were compared (Figure 7, Relative inhibitory potencies (R.I.P.) are valency-corrected and calculated according as: $\left.\frac{I C_{50, \text { monovalent }}}{I C_{50, \text { multivalent }} \times \text { valency }}\right)$.

Comparing constructs of the same valency, glycodendrimers bearing the monovalent ligand $\mathbf{3}$ had lower $\mathrm{IC}_{50}$ values than the ones carrying 1 , and all of them were more active than the simply mannosylated analogs (Figure 7). Once again, dendrimers based on 2 behaved similarly to the corresponding derivatives of 1 ; moreover, the tetravalent and the hexavalent compounds $\mathbf{1 0 . 2}$ and $\mathbf{1 1 . 2}$ showed a R.I.P. $<$ 1. This confirmed that the high activity of the monovalent ligand $\mathbf{2}$ is due to an outperforming binding mode not allowed for its multivalent derivatives. On the contrary, the activity of the multivalent presentations of compounds $\mathbf{1}$ and 3 gradually increased by incrementing the valency. Remarkably, R.I.P. values obtained for dendrimers based on $\mathbf{3}$ grew faster with valency than those shown by the corresponding dendrimers based on $\mathbf{1}$ or on mannose (Figure 7). These results highlight that affinity differences at the monovalent level are amplified by multivalent presentation and support the importance of optimizing the structure of monovalent ligands. Tetravalent $\mathbf{1 0 . 1}$ and $\mathbf{1 0 . 2}$ had an analogous affinity for DC-SIGN with respect to 4.1 and 4.2 , suggesting that, being the 
valency equal, the shape of this type of dendrimers does not influence their activity to a significant extent. .

Compounds 10.1, 10.3 and 11.3 were tested also in cellular infection models, by evaluating their ability to inhibit the DC-SIGN-mediated HIV infection of $\mathrm{CD}^{+}{ }^{+}$T-cells. Also in this case, the activity of the monovalent ligand had a positive role, since $\mathbf{1 0 . 3}$ was more active than 10.1. The importance of the valency was also confirmed: the hexavalent compound $\mathbf{1 1 . 3}$ was around 10 times more active than the tetravalent 10.3. Remarkably, despite the good results shown by glycodendrimers bearing mimic 3 , a further valency increase, as in 13.9.3 (Figure 6), was not practical, due to low water solubility of the constructs.

Molecular dynamic simulations were used to estimate the maximum distance spanned by dendrimers $\mathbf{1 0 . 1}$ and $\mathbf{1 1 . 1}$ (as simpler models of $\mathbf{1 0 . 3}$ and 11.3, respectively), which in turn allowed to dissect the possible mechanisms of interaction between the glycodendrimers and DC-SIGN. Since the calculated maximum extension of $\mathbf{1 1 . 1}$ was $35.4 \AA$ and the distance between two contiguous DC-SIGN binding sites in one tetramer is at least $38 \AA^{28}$, the chelation mechanism was excluded. Remarkably, the hexavalent compound $\mathbf{1 1 . 1}$ had an $\mathrm{IC}_{50}$ value comparable to that of 12.9.1, which is also hexavalent, but is built on a longer PEG core, suggesting that a flexible linker is not suitable to make the active ligands assuming the proper conformation for chelation.

\section{Phenylene-ethynylene rod-like scaffolds}

With the goal of further improving dendrimers activity by exploiting the chelation mechanism, the length and the rigidity of the scaffolds were optimized. In a collaboration with the group of Roland J. Pieters (Utrecht University), three phenylene-ethynylene rod-like spacers of different lengths (i.e. 8, 12.5 and $22 \AA$ ) were synthesized through multiple Sonogashira reactions and deprotection steps ${ }^{29}$. Their terminal triple bonds were exploited to 
connect the glycomimetic moieties by $\mathrm{CuAAC}$ reactions. To this aim, trivalent dendrons 9.1 and 9.3 were employed, as well as the monovalent ligands $\mathbf{1}$ and $\mathbf{3}$ and their elongated versions, characterized by a PEG linker having the same length of the dendron chain ${ }^{30}$. The structures of the obtained hexavalent dendrimers $(14.9 .1,15.9 .1,16.9 .1,14.9 .3,15.9 .3$, 16.9.3) and divalent controls $(16.1,16.3$ and $14.1 \mathrm{~L}, 15.1 \mathrm{~L}, 16.1 \mathrm{~L}, 14.3 \mathrm{~L}, 15.3 \mathrm{~L}, 16.3 \mathrm{~L})$ are depicted in Figure 8.

Molecular dynamics simulations showed that all these constructs have the potential to chelate two contiguous DC-SIGN binding sites: at maximum extension both the shortest dendrimer 14.9.1 and the longest one 16.9.1 are longer than $4 \mathrm{~nm}$. As before, SPR competition assays were performed to measure their $\mathrm{IC}_{50}$ and R.I.P. values (Figure 9). The hexavalent derivatives of $3(14.9 .3,15.9 .3$ and 16.9.3) could not be evaluated appropriately in this format, because their affinity reached the lower limit of the assay (Figure 9). Nonetheless, valuable information could be gathered concerning the effect of the spacers on the dendrimers' activity.

As expected, compounds based on the more active ligand $\mathbf{3}$ outperformed those based on $\mathbf{1}$ (see, for instance, divalent $\mathbf{1 6 . 1}$ and 16.3). The bioactivity was also clearly dependent on the valency, since hexavalent compounds were more active than the corresponding divalent ones (compare 16.9.1 and 16.1), demonstrating the positive effect of increasing the local ligand concentration. Entropic penalties were paid by the structures characterized by the flexible PEG linker (1.L and 3.L derivatives); indeed, $\mathbf{1 6 . 1}$ and $\mathbf{1 6 . 3}$ were about two times more active than 16.1.L and 16.3.L, respectively. Finally, $\mathrm{IC}_{50} \mathrm{~s}$ decreased regularly with the length of the rod, suggesting that the chelation mechanism may become increasingly accessible.

For the hexavalent derivatives of $\mathbf{3}$ a more sensitive cellular HIV infection study was performed, revealing again a clear dependency on the length of the rod core. In particular, the bioactivity increased by about one order of magnitude from 11.3 to 14.9 .3 , by simply 
inserting the shortest rod as the dendrimer core, and it was highest for the longest 16.9.3. The valency effect was also confirmed, since hexavalent compounds were more active than the divalent ones. $\mathrm{IC}_{50}$ values were measured for 14.9.3, 16.9.3, 16.3 as $67 \mathrm{nM}, 24 \mathrm{nM}$ and 161 $\mathrm{nM}$, respectively. To the best of our knowledge, 16.9.3 is one of the most potent inhibitors of the DC-SIGN-mediated trans HIV infection described up to date, despite its relatively moderate valency and strengthened by its controlled and well characterized structure. This result clearly highlights how the combination of optimized monovalent ligands with finetuned multivalent constructs of controllable size can maximize the effect of valency when targeting a multivalent receptor.

Remarkably, even if these dendrimer-like compounds, bearing a lipophilic core and hydrophilic carbohydrate groups, are endowed with amphiphilic properties, they do not significantly aggregate in aqueous solution, as shown using Dynamic Light Scattering, Analytical Ultracentrifugation and a number of other biophysical techniques ${ }^{31}$. Hence, their performances in infection studies can be correctly interpreted based on their individual structure.

\section{Biological characterization}

Three of the dendrimers described above, $4.2^{32}, 11.3^{33}$ and $16.9 .3^{34}$, underwent further biological assays to achieve a fuller characterization of their effect. Importantly, none of the compounds was found to be toxic against the tested cell lines, and both 4.2 and $\mathbf{1 1 . 3}$ did not alter the viability of human cervical explants up to a concentration of $1 \mathrm{mM}$.

In particular, dendron 4.2 and dendrimer 11.3 were tested as inhibitors of HIV infection on explants obtained from human uterine cervix, to achieve a better approximation of in vivo conditions. To this aim, tissue explants were exposed to different types of R5 tropic HIV-1, including the laboratory adapted $\mathrm{BaL}$ and clinical primary isolates, in the presence of 


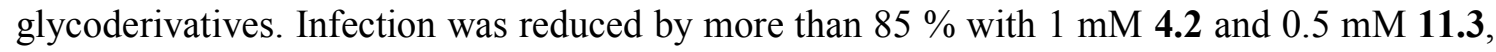
demonstrating that these inhibitors are good precursors to be further optimized and formulated as microbicide drugs.

It is well-known that the DC-SIGN binding to a ligand can activate and regulate the immune response $^{35}$, therefore DC-SIGN binders are also studied for the development of vaccines and immunomodulants. The activation of DC-SIGN signaling by the dendrimers synthesized in our group was investigated to assess if they stimulate the production of factors able to interfere with HIV infection and if they are suitable as immunomodulants and vaccine adjuvants. In this study, the expression of cytokines, chemokines and genes involved in immune responses was evaluated after immature Monocyte Derived Dendritic Cells (iMDDCs) interaction with DC-SIGN antagonists. Preliminarily, the behavior of $\mathbf{4 . 2}$ was explored $^{32}$, showing that it induced an increase of the production of $\beta$ chemokines known to suppress HIV-1 R5 tropic strain replication ${ }^{36}$. A similar activity was observed for $\mathbf{1 1 . 3}^{33}$ and 16.9.3 ${ }^{34}$, that also stimulated an impressive production of antiviral and pro-inflammatory cytokines, as well as of co-stimulatory molecules having a role in the activation of naïve $\mathrm{T}$ cells. Additional cytokines and proteins that promote Dendritic Cells differentiation, development, survival and activation were overexpressed by treating iMDDCs with 16.9.3.

Although a direct comparison between the effects of the tested compounds could not be drawn, since experiments were conducted on different healthy donors, using different techniques and experimental settings, it was noticed that the inflammatory response stimulated by $\mathbf{1 6 . 9 . 3}$ was less pronounced than that caused by 8.3. This is an important element to acquire, since an excessive immune response can result in tissue damage (and even death). It was therefore concluded that these compounds and, in particular, the most active DC-SIGN antagonist 16.9.3, have a potential use as adjuvants and immunostimulants in the 
304 formulation of vaccines for HIV and, generally, mucosal pathogens. Further studies in both ex 305 vivo and in vivo models will be required.

306 Exploiting the intrinsic fluorescence of its rod core, the cellular uptake and internalization 307 route of 16.9.3 within human iMDDCs was investigated by means of fluorescence 308 microscopy at varying time and temperature intervals ${ }^{34}$. It was observed that $\mathbf{1 6 . 9 . 3}$ is 309 internalized by DCs already after $10 \mathrm{~min}$ of incubation at room temperature, but not at $4{ }^{\circ} \mathrm{C}$, 310 an indication that uptake occurs through an energy-dependent mechanism, such as the 311 receptor-mediated one.

312 Finally, confocal images with fluorescent tracers used to mark specific sub-cellular organelles 313 revealed that $\mathbf{1 6 . 9 . 3}$ is internalized mainly to lysosomes and that it also transits through and 314 resides in early endosomes for a quite prolonged period of time. These pulse-chase 315 experiments showed that the dendrimer uptake is rapid and confirmed that the molecule has 316 the potential to elicit an immune response that may be exploited for the development of 317 vaccines.

\section{Summary and outlook}

We have here presented the results of our search for efficient antagonists of DC-SIGN, a C-

321 type lectin recognizing highly mannosylated structures, and involved in the recognition

322 process of pathogens such as HIV, Ebola and Dengue viruses. Rather than directly using mannose- or oligomannose derivatives, our strategy has first been devoted to the optimization of glycomimetic monovalent DC-SIGN ligands. We have then combined the most promising monovalent ligands with several types of dendrimer-like scaffolds, to produce multivalent materials targeted against C-type lectins and in particular DC-SIGN. The activity trend of the

327 monovalent ligands was reflected in the bioactivity of the dendrimers, thus highlighting the 328 importance of ligand optimization. Also the valency influenced dendrimers activity; indeed, 
among each dendrimer sub-class, the activity generally increased by increasing the valency. The size of the dendrimers was finally tuned using rigid rod-like cores of controlled length, in order to control the relative orientation and the distance spanned by the active ligands. In this way, we synthesized a class of dendrimer-like molecules that can reach simultaneously two adjacent DC-SIGN binding sites within the tetramer carrying to each site a small cluster of glycomimetic ligands. These systems, thus, can exploit more than one multivalency mechanism (e.g. chelation, statistical rebinding), which results in a major potency enhancement compared to shorter or more flexible constructs. With an appropriate combination of monovalent ligands, rod-core and valency, the hexavalent compound 16.9.3, one of the most potent inhibitors of the DC-SIGN-mediated HIV infection up to date, was obtained. This result showed how the rational design of artificial ligands helps in obtaining powerful compounds while retaining a moderate valency, which allows to deal with perfectly monodisperse and well-characterized species. DC-SIGN antagonists such as $\mathbf{1 6 . 9 . 3}$ are effective precursors for further derivatizations and appropriate formulations, with the aim of producing topical microbicides as pathogen-entry inhibitors. Moreover, we have observed that 16.9.3 is internalized by dendritic cells and routed to endolysosomal compartments. These features, together with its ability to induce the overexpression of signaling molecules involved in immune responses, make 16.9.3 interesting as an immonomodulator and vaccine adjuvant.

\section{Acknowledgements}

The research described in this review was carried out with support from the European Union through Marie-Curie program ETN-CARMUSYS (PITN-GA-2008-213592) and COST Action CM1102 MultiGlycoNano. The fellowships of S.O. and G.G. were funded by the University of Milan. 
354

355

356

357

358

359

360

361

362

363

364

365

366

367

368

369

370

371

372

373

374

375

376

377

378

\section{References}

(1) www.unaids.org Web. Accessed on 10 Jan 2017.

(2) Wang, H. B.; Mo, Q. H.; Yang, Z. J. Immunol. Res. 2015, 2015, 503978.

(3) Pan, X.; Baldauf, H. M.; Keppler, O. T.; Fackler, O. T. Cell Res. 2013, 23, 876.

(4) Szunerits, S.; Barras, A.; Khanal, M.; Pagneux, Q.; Boukherroub, R. Molecules 2015, $20,14051$.

(5) Sepulveda-Crespo, D.; Cena-Diez, R.; Jimenez, J. L.; Angeles Munoz-Fernandez, M. Med. Res. Rev. 2017, 37, 149.

(6) Gringhuis, S. I.; den Dunnen, J.; Litjens, M.; van Het Hof, B.; van Kooyk, Y.; Geijtenbeek, T. B. Immunity 2007, 26, 605.

(7) van Kooyk, Y.; Geijtenbeek, T. B. Nat. Rev. Immunol. 2003, 3, 697.

(8) Tabarani, G.; Reina, J. J.; Ebel, C.; Vives, C.; Lortat-Jacob, H.; Rojo, J.; Fieschi, F. FEBS Lett. 2006, 580, 2402.

(9) Reina, J. J.; Rojo, J. Braz. J. Pharm. Sci. 2013, 49, 109.

(10) Sattin, S.; Bernardi, A. Trends Biotechnol. 2016, 34, 483.

(11) Adams, E. W.; Ratner, D. M.; Bokesch, H. R.; McMahon, J. B.; O'Keefe, B. R.; Seeberger, P. H. Chem. Biol. 2004, 11, 875.

(12) Shahzad-Ul-Hussan, S.; Sastry, M.; Lemmin, T.; Soto, C.; Loesgen, S.; Scott, D. A.; Davison, J. R.; O'Connor, R.; Kwong, P. D.; Bewley, C. A. ChemBioChem 2017.

(13) Mari, S.; Posteri, H.; Marcou, G.; Potenza, D.; Micheli, F.; Cañada, F. J.; JimenezBarbero, J.; Bernardi, A. Eur. J. Org. Chem. 2004, 2004, 5119.

(14) Reina, J. J.; Sattin, S.; Invernizzi, D.; Mari, S.; Martinez-Prats, L.; Tabarani, G.; Fieschi, F.; Delgado, R.; Nieto, P. M.; Rojo, J.; Bernardi, A. ChemMedChem 2007, 2, 1030.

(15) Mari, S.; Sanchez-Medina, I.; Mereghetti, P.; Belvisi, L.; Jimenez-Barbero, J.; Bernardi, A. Carbohydr. Res. 2007, 342, 1859. 
(16) Sattin, S.; Daghetti, A.; Thepaut, M.; Berzi, A.; Sanchez-Navarro, M.; Tabarani, G.; Rojo, J.; Fieschi, F.; Clerici, M.; Bernardi, A. ACS Chem. Biol. 2010, 5, 301.

(17) Thepaut, M.; Guzzi, C.; Sutkeviciute, I.; Sattin, S.; Ribeiro-Viana, R.; Varga, N.; Chabrol, E.; Rojo, J.; Bernardi, A.; Angulo, J.; Nieto, P. M.; Fieschi, F. J. Am. Chem. Soc. 2013, 135, 2518.

(18) Sutkeviciute, I.; Thepaut, M.; Sattin, S.; Berzi, A.; McGeagh, J.; Grudinin, S.; Weiser, J.; Le Roy, A.; Reina, J. J.; Rojo, J.; Clerici, M.; Bernardi, A.; Ebel, C.; Fieschi, F. ACS Chem. Biol. 2014, 9, 1377.

(19) Varga, N.; Sutkeviciute, I.; Guzzi, C.; McGeagh, J.; Petit-Haertlein, I.; Gugliotta, S.; Weiser, J.; Angulo, J.; Fieschi, F.; Bernardi, A. Chem. - Eur. J. 2013, 19, 4786.

(20) Lundquist, J. J.; Toone, E. J. Chem. Rev. 2002, 102, 555.

(21) Pieters, R. J. Org. Biomol. Chem. 2009, 7, 2013.

(22) Renaudet, O.; Roy, R. Chem. Soc. Rev. 2013, 42, 4515.

(23) Chabre, Y. M.; Roy, R. Adv. Carbohydr. Chem. Biochem. 2010, 63, 165.

(24) Luczkowiak, J.; Sattin, S.; Sutkeviciute, I.; Reina, J. J.; Sanchez-Navarro, M.; Thepaut, M.; Martinez-Prats, L.; Daghetti, A.; Fieschi, F.; Delgado, R.; Bernardi, A.; Rojo, J. Bioconjug. Chem. 2011, 22, 1354.

(25) Goti, G.; Palmioli, A.; Stravalaci, M.; Sattin, S.; De Simoni, M. G.; Gobbi, M.; Bernardi, A. Chem. - Eur. J. 2016, 22, 3686.

(26) Gesuete, R.; Storini, C.; Fantin, A.; Stravalaci, M.; Zanier, E. R.; Orsini, F.; Vietsch, H.; Mannesse, M. L.; Ziere, B.; Gobbi, M.; De Simoni, M. G. Ann. Neurol. 2009, 66, 332.

(27) Varga, N.; Sutkeviciute, I.; Ribeiro-Viana, R.; Berzi, A.; Ramdasi, R.; Daghetti, A.; Vettoretti, G.; Amara, A.; Clerici, M.; Rojo, J.; Fieschi, F.; Bernardi, A. Biomaterials 2014, 35,4175 . 
403

404

405

406

407

408

409

410

411

412

413

414

415

416

417

418

419

420

421

422

423

424

(28) Tabarani, G.; Thepaut, M.; Stroebel, D.; Ebel, C.; Vives, C.; Vachette, P.; Durand, D.;

Fieschi, F. J. Biol. Chem. 2009, 284, 21229.

(29) Pertici, F.; Varga, N.; van Duijn, A.; Rey-Carrizo, M.; Bernardi, A.; Pieters, R. J. Beilstein J. Org. Chem. 2013, 9, 215.

(30) Ordanini, S.; Varga, N.; Porkolab, V.; Thepaut, M.; Belvisi, L.; Bertaglia, A.; Palmioli, A.; Berzi, A.; Trabattoni, D.; Clerici, M.; Fieschi, F.; Bernardi, A. Chem. Commun. 2015, 51, 3816 .

(31) Ordanini, S.; Zanchetta, G.; Porkolab, V.; Ebel, C.; Fieschi, F.; Guzzetti, I.; Potenza, D.; Palmioli, A.; Podlipnik, C.; Meroni, D.; Bernardi, A. Macromol. Biosci. 2016, 16, 896.

(32) Berzi, A.; Reina, J. J.; Ottria, R.; Sutkeviciute, I.; Antonazzo, P.; Sanchez-Navarro, M.; Chabrol, E.; Biasin, M.; Trabattoni, D.; Cetin, I.; Rojo, J.; Fieschi, F.; Bernardi, A.; Clerici, M. AIDS 2012, 26, 127.

(33) Berzi, A.; Varga, N.; Sattin, S.; Antonazzo, P.; Biasin, M.; Cetin, I.; Trabattoni, D.; Bernardi, A.; Clerici, M. Viruses 2014, 6, 391.

(34) Berzi, A.; Ordanini, S.; Joosten, B.; Trabattoni, D.; Cambi, A.; Bernardi, A.; Clerici, M. Sci. Rep. 2016, 6, 35373.

(35) Svajger, U.; Anderluh, M.; Jeras, M.; Obermajer, N. Cell. Signalling 2010, 22, 1397.

(36) Cocchi, F.; DeVico, A. L.; Garzino-Demo, A.; Arya, S. K.; Gallo, R. C.; Lusso, P. Science 1995, 270, 1811. 


\section{$425 \quad$ Figure captions}

426 Figure 1: Chemical structure of $(\mathrm{Man})_{9}(\mathrm{GlcNAc})_{2}$ glycan; the disaccharide Man $\alpha 1-2 \mathrm{Man}$ and

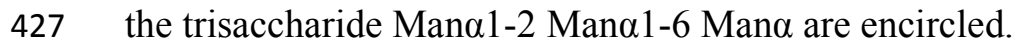

428 Figure 2: Structure of the synthesized pseudo-mannosylated compounds: a) pseudo429 disaccharide 1 mimics the Man $\alpha$ 1-2Man disaccharide; b) pseudo-trisaccharide 2 mimics the

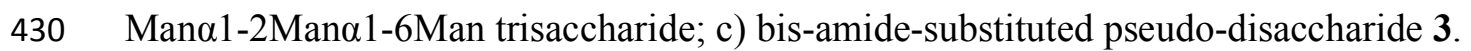
Figure 3: Possible affinity-enhacement mechanisms occurring between a multivalent ligand (black) and a multivalent protein (gray): a) chelation; b) statistical rebinding; c) protein aggregation.

Figure 4: Structure of scaffolds $\mathbf{4}$ and $\mathbf{5}$ and the corresponding tetravalent $\mathbf{4 . 1}$ and $\mathbf{4 . 2}$ 435 glycosylated dendrons and 32-valent glycosylated $\mathbf{5 . 1}$ and $\mathbf{5 . 2}$ dendrimers.

Figure 5: Structure of tetravalent dendrons $\mathbf{4 N . 1}$ and $\mathbf{4 N . 2}$, stabilized by replacing the succinyl ester bond with an amido bond.

Figure 6: Structure of the glycodendrimers based on monovalent mannose, 1, 2 or 3 and multivalent polyalkyne terminated scaffolds.

440 Figure 7: $\mathrm{IC}_{50}$ (empty white bars) and relative inhibitory potency (R.I.P.) (black bars) values 441 of 1, 2, 3 and mannose multivalent derivatives, tested as DC-SIGN binders through SPR 442 competition assays.

Figure 8: Structure of the glycodendrimers based on monovalent ligands $\mathbf{1}$ or $\mathbf{3}$ and rigid phenylene-ethynylene scaffolds.

Figure 9: $\mathrm{IC}_{50}$ (empty white bars) and relative inhibitory potency (R.I.P.) (black bars) values

446 of rod-based derivatives of $\mathbf{1}$ and $\mathbf{3}$, tested as DC-SIGN binders by SPR competition assays.

447 Scheme 1: Synthesis of tetramine 6 and of the tetravalent dendron $4 \mathbf{N}$ 


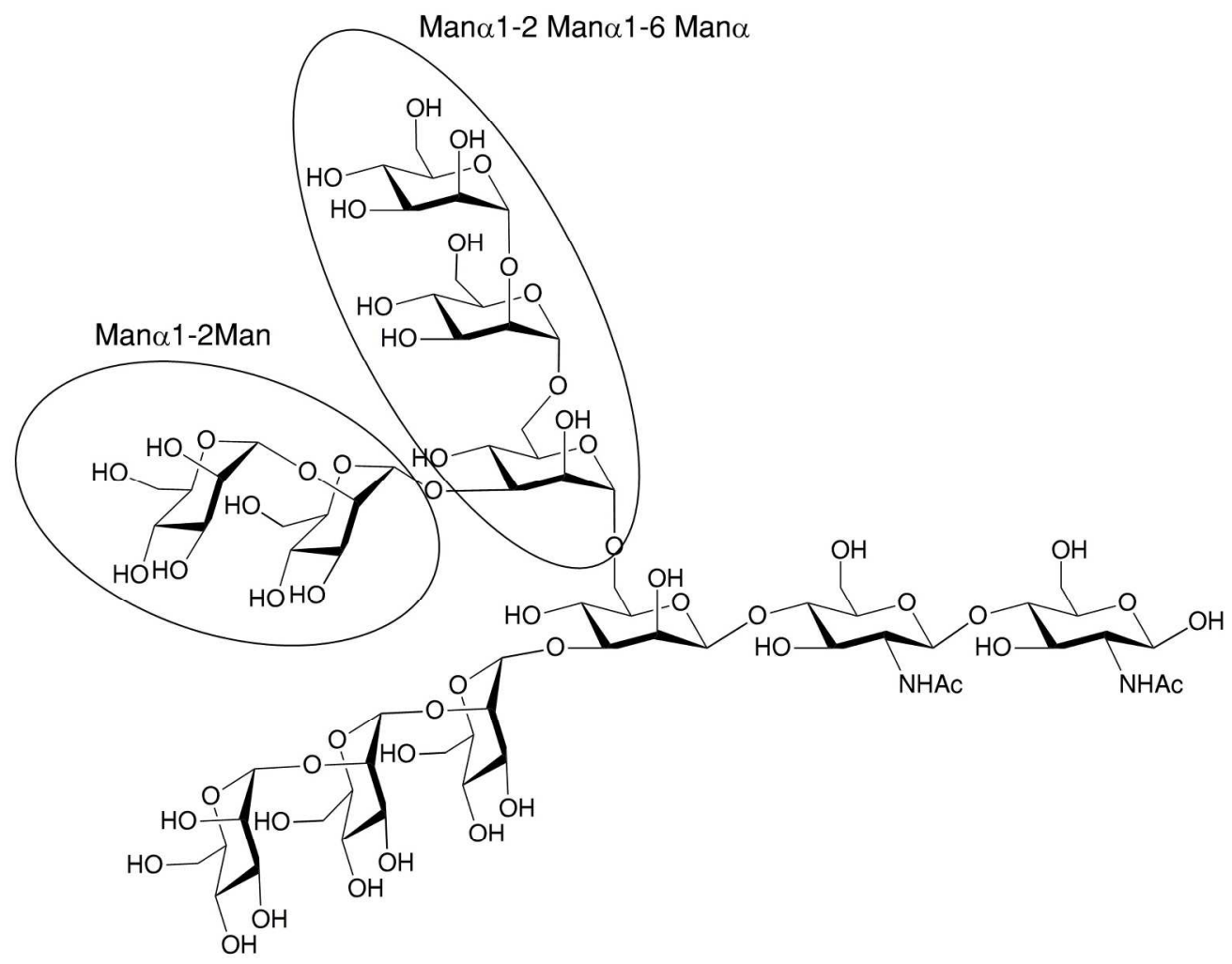

Figure 1: Chemical structure of (Man $)_{9}(\mathrm{GlcNAc})_{2}$ glycan; the disaccharide Mana1-2Man and the trisaccharide Mana1-2 Mana1-6 Mana are encircled.

$184 \times 144 \mathrm{~mm}(300 \times 300 \mathrm{DPI})$ 


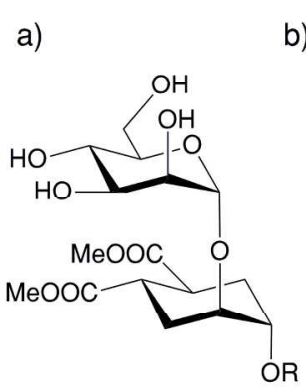

1 b)

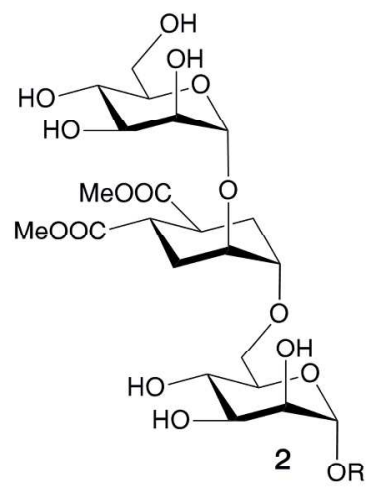

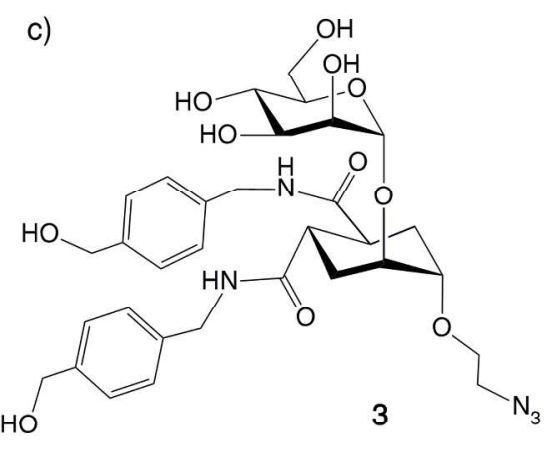

a $\mathrm{R}=-\mathrm{CH}_{2} \mathrm{CH}_{2} \mathrm{NH}_{2}$

Figure 2: Structure of the synthesized pseudo-mannosylated compounds: a) pseudo-disaccharide $\mathbf{1}$ mimics the Mana1-2Man disaccharide; b) pseudo-trisaccharide 2 mimics the Mana1-2Mana1-6Man trisaccharide; c) bis-amide-substituted pseudo-disaccharide $\mathbf{3}$.

$191 \times 84 \mathrm{~mm}(300 \times 300 \mathrm{DPI})$ 
a)

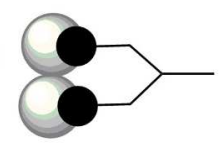

b)

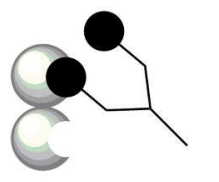

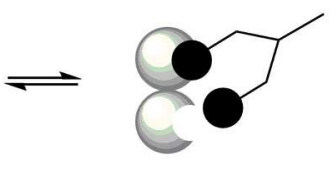

c)

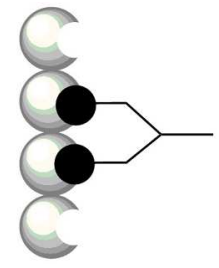

Figure 3: Possible affinity-enhacement mechanisms occurring between a multivalent ligand (black) and a multivalent protein (gray): a) chelation; b) statistical rebinding; c) protein aggregation.

$186 \times 37 \mathrm{~mm}(300 \times 300 \mathrm{DPI})$ 

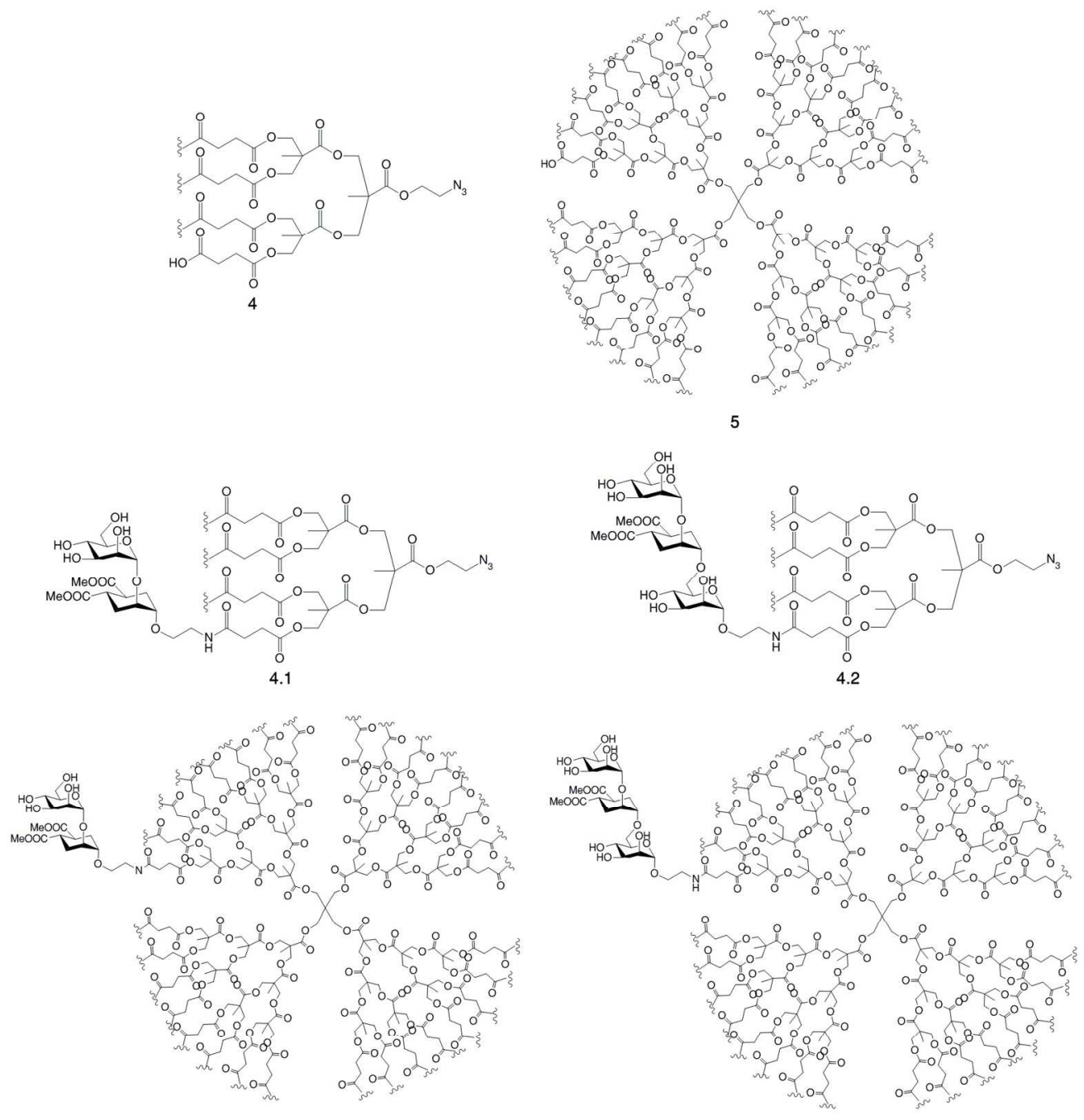

5.1

5.2

Figure 4: Structure of scaffolds $\mathbf{4}$ and $\mathbf{5}$ and the corresponding tetravalent $\mathbf{4 . 1}$ and $\mathbf{4 . 2}$ glycosylated dendrons and 32-valent glycosylated $\mathbf{5 . 1}$ and $\mathbf{5 . 2}$ dendrimers.

$157 \times 167 \mathrm{~mm}(300 \times 300$ DPI $)$ 

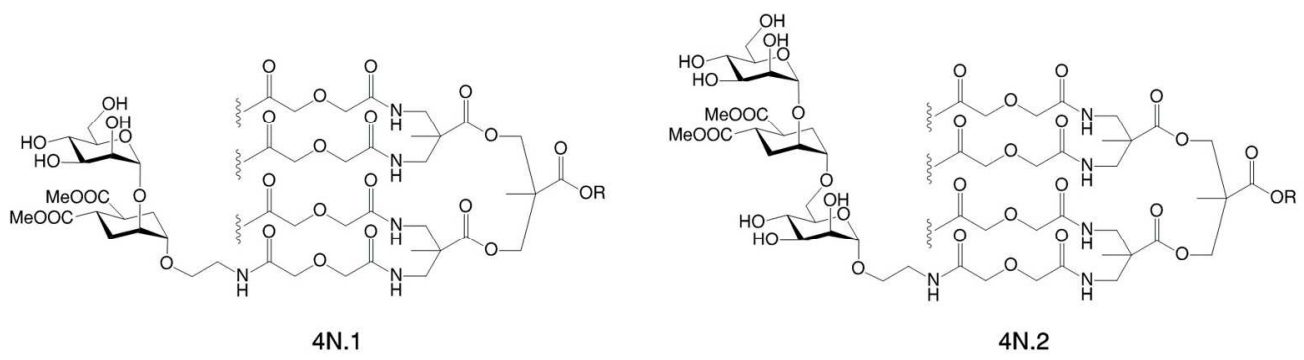

Figure 5: Structure of tetravalent dendrons $\mathbf{4 N . 1}$ and $\mathbf{4 N . 2}$, stabilized by replacing the succinyl ester bond with an amido bond.

$156 \times 42 \mathrm{~mm}(300 \times 300$ DPI $)$ 


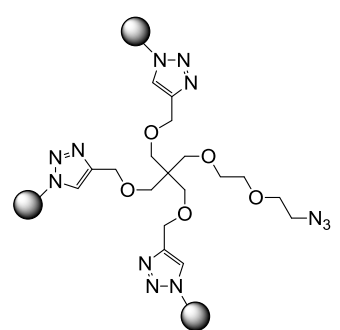

9.Man if $\mathrm{O}=$ mannose

9.1 if $O=1$

9.3 if $O=3$

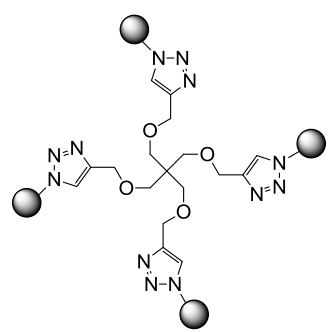

10.Man if $\mathrm{O}=$ mannose

10.1 if $\mathrm{O}=1$

10.2 if $O=2$

10.3 if $\mathrm{O}=\mathbf{3}$

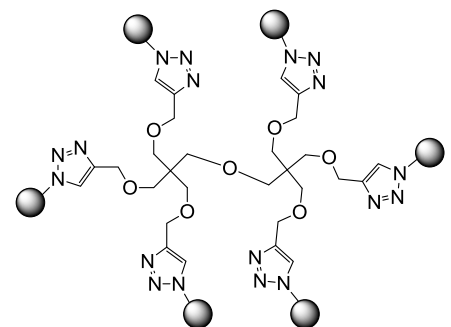

11. Man if $\mathrm{O}=$ mannose

11.1 if $O=1$

11.2 if $\mathrm{O}=2$

11.3 if $O=3$
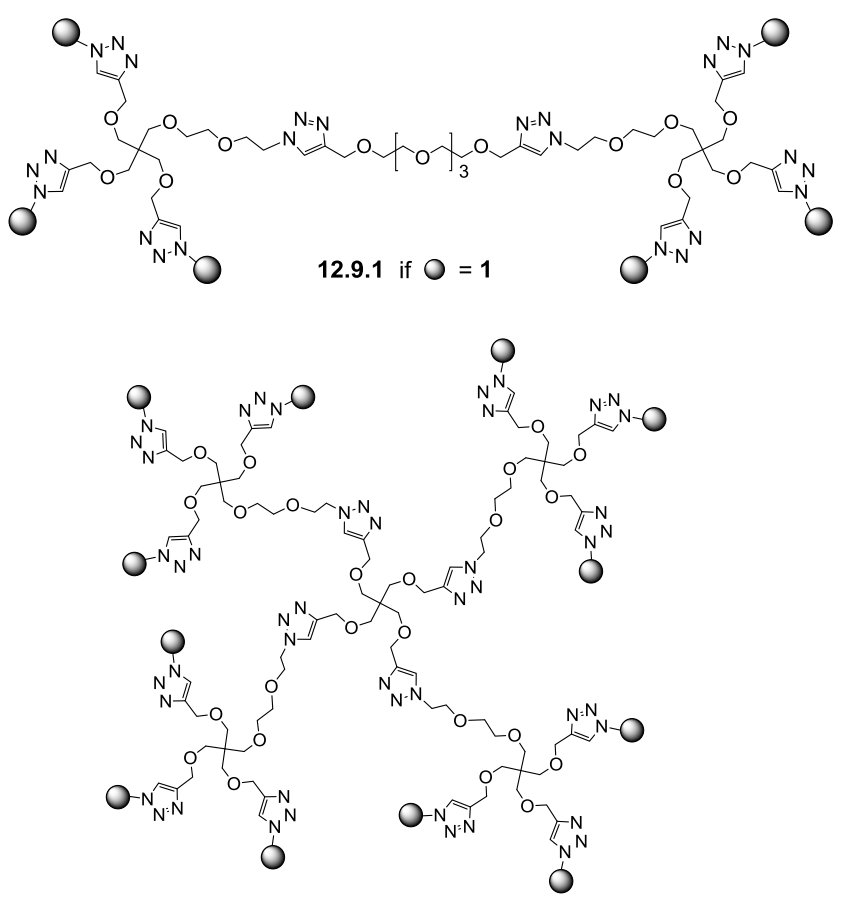

10.9. Man if $\bigcirc=$ mannose
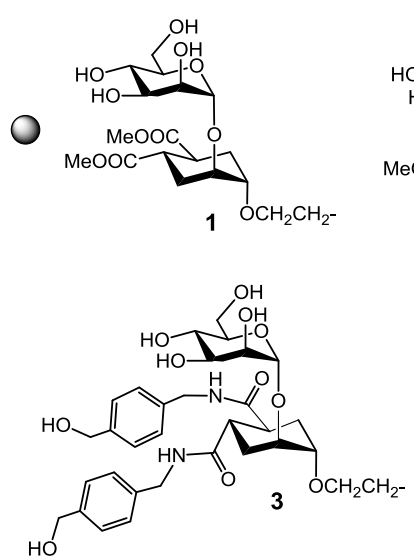
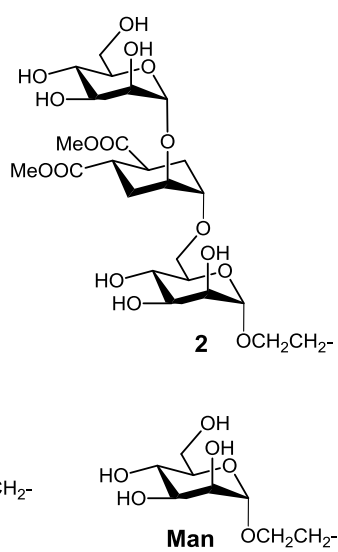

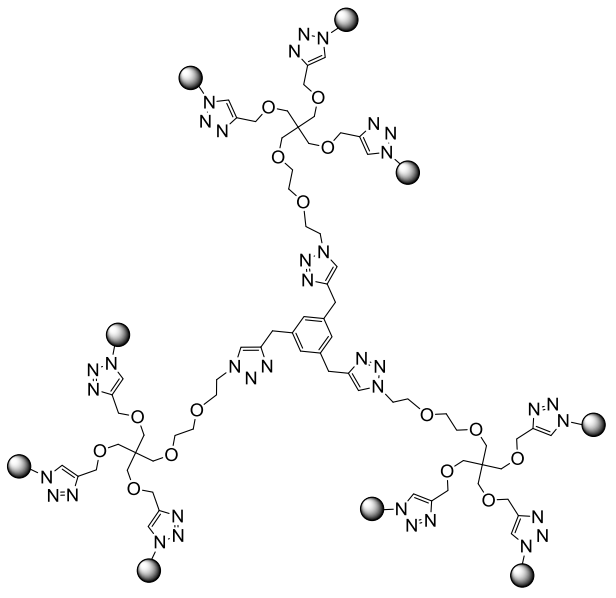

13.9. Man if $O=$ mannose

13.9.1 if $\mathrm{O}=1$

13.9.3 if $\mathrm{O}=3$

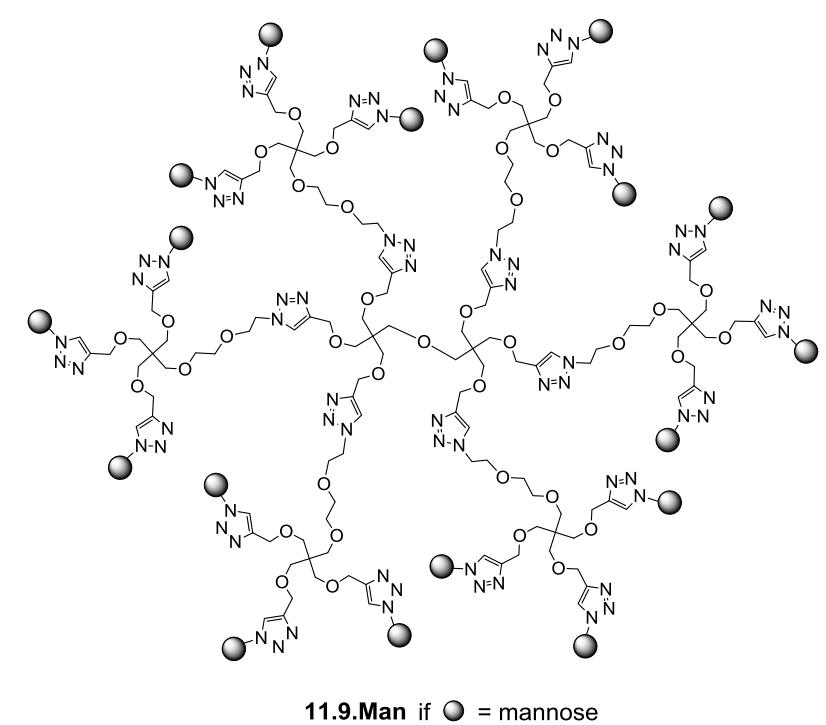




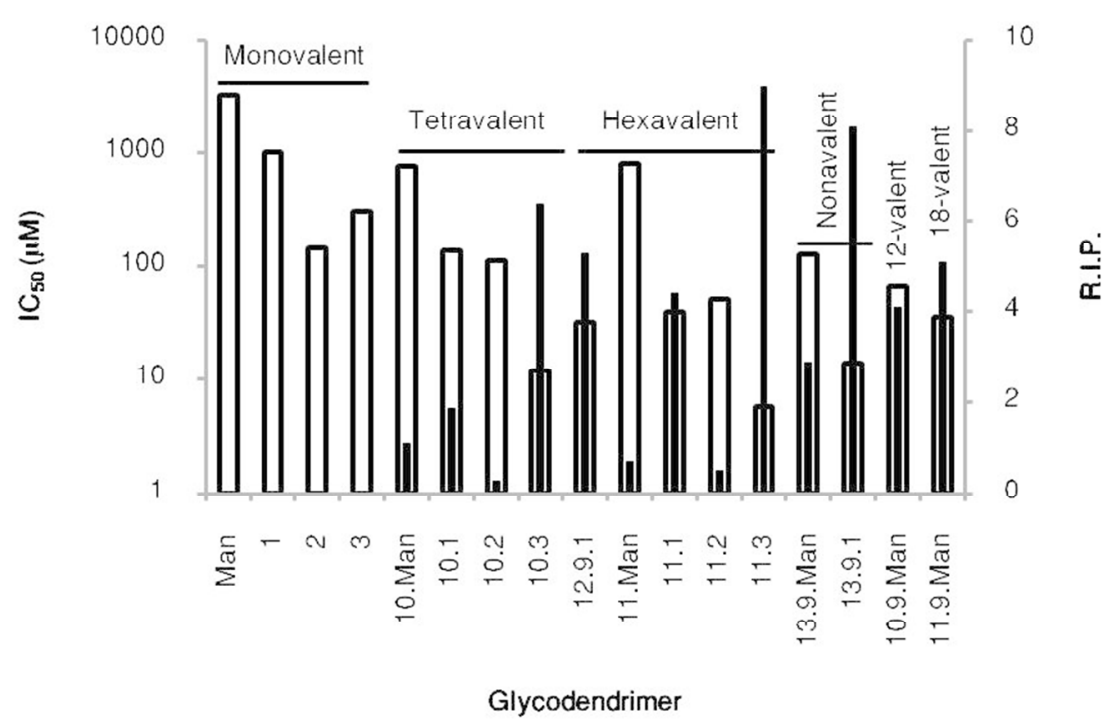

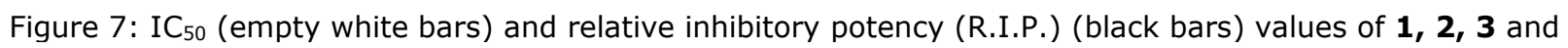
mannose multivalent derivatives, tested as DC-SIGN binders through SPR competition assays.

$86 \times 49 \mathrm{~mm}(300 \times 300 \mathrm{DPI})$ 

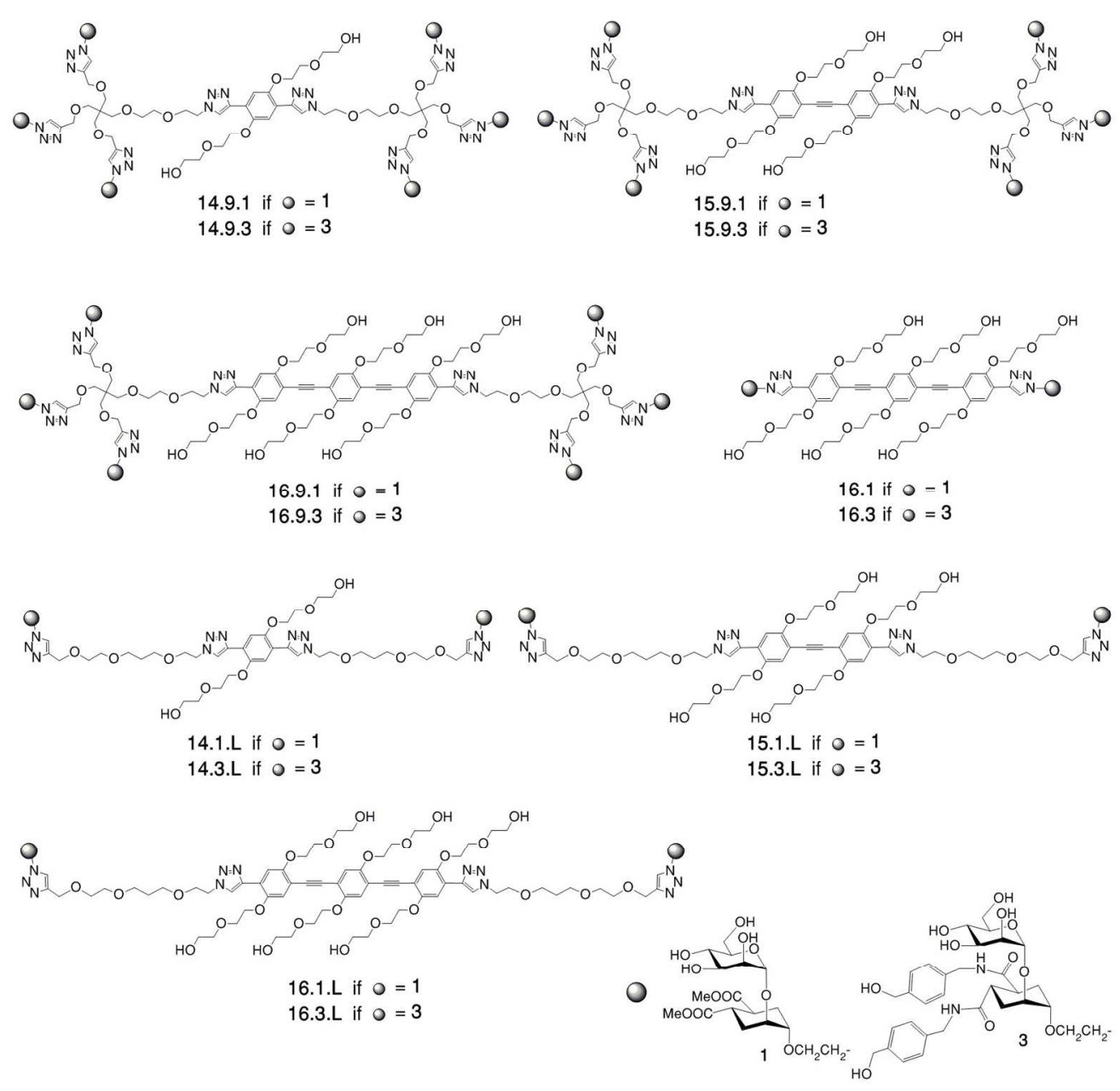

Figure 8: Structure of the glycodendrimers based on monovalent ligands $\mathbf{1}$ or $\mathbf{3}$ and rigid phenyleneethynylene scaffolds.

$152 \times 147 \mathrm{~mm}(300 \times 300 \mathrm{DPI})$ 


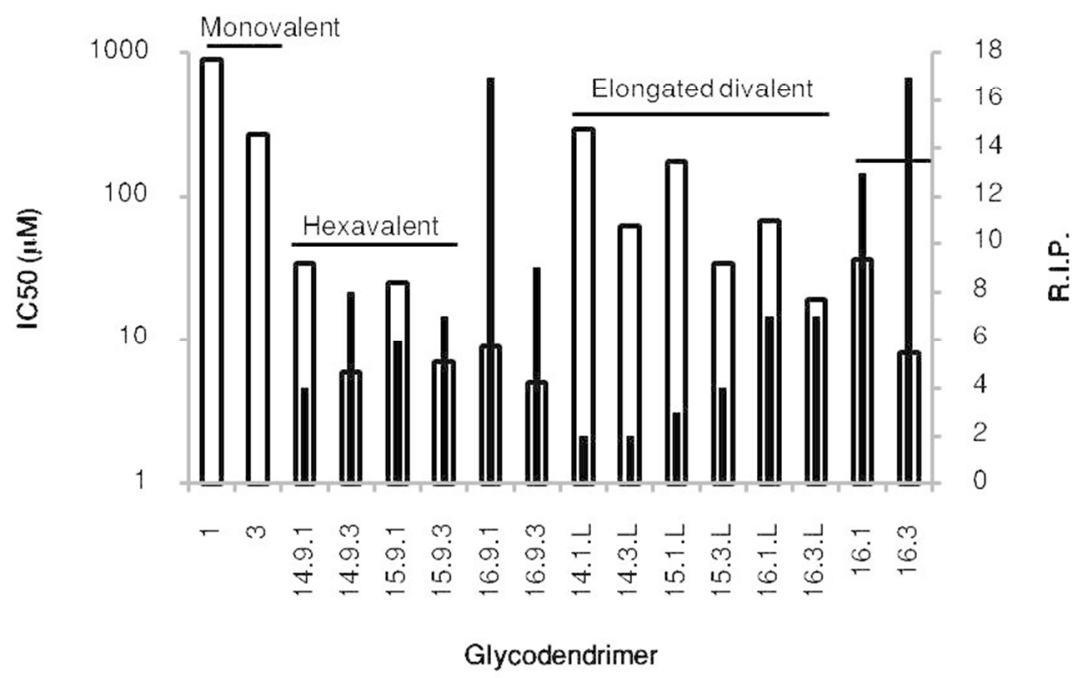

Figure 9: IC $_{50}$ (empty white bars) and relative inhibitory potency (R.I.P.) (black bars) values of rod-based derivatives of $\mathbf{1}$ and $\mathbf{3}$, tested as DC-SIGN binders by SPR competition assays.

$86 \times 49 \mathrm{~mm}(300 \times 300 \mathrm{DPI})$ 


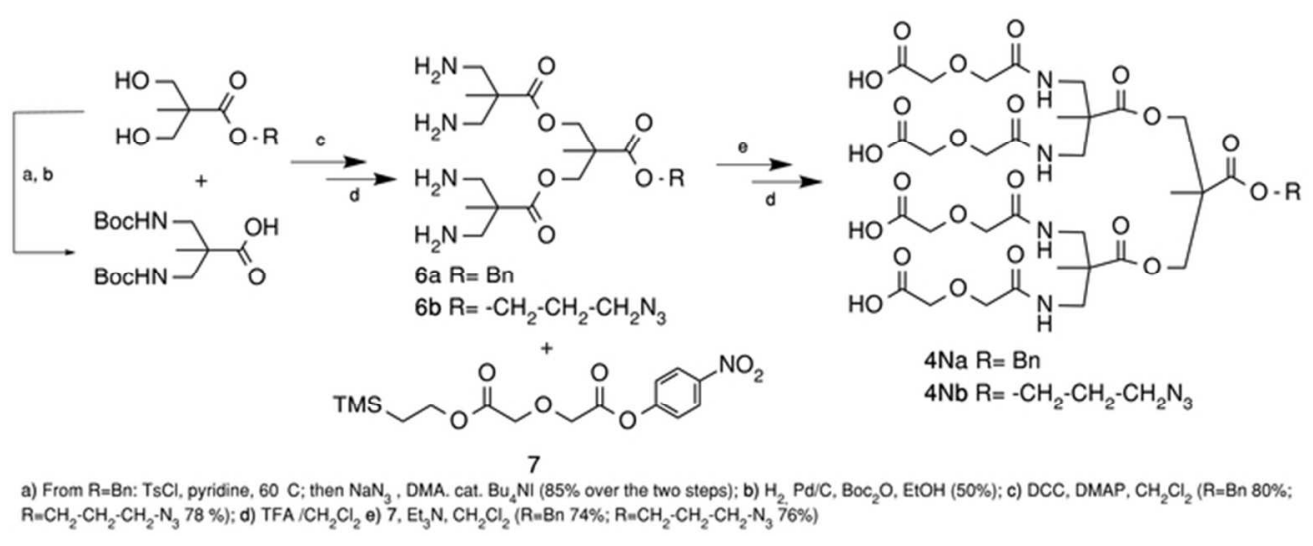

Scheme 1: Synthesis of tetramine $\mathbf{6}$ and of the tetravalent dendron $\mathbf{4 N}$

$60 \times 24 \mathrm{~mm}(300 \times 300$ DPI $)$ 


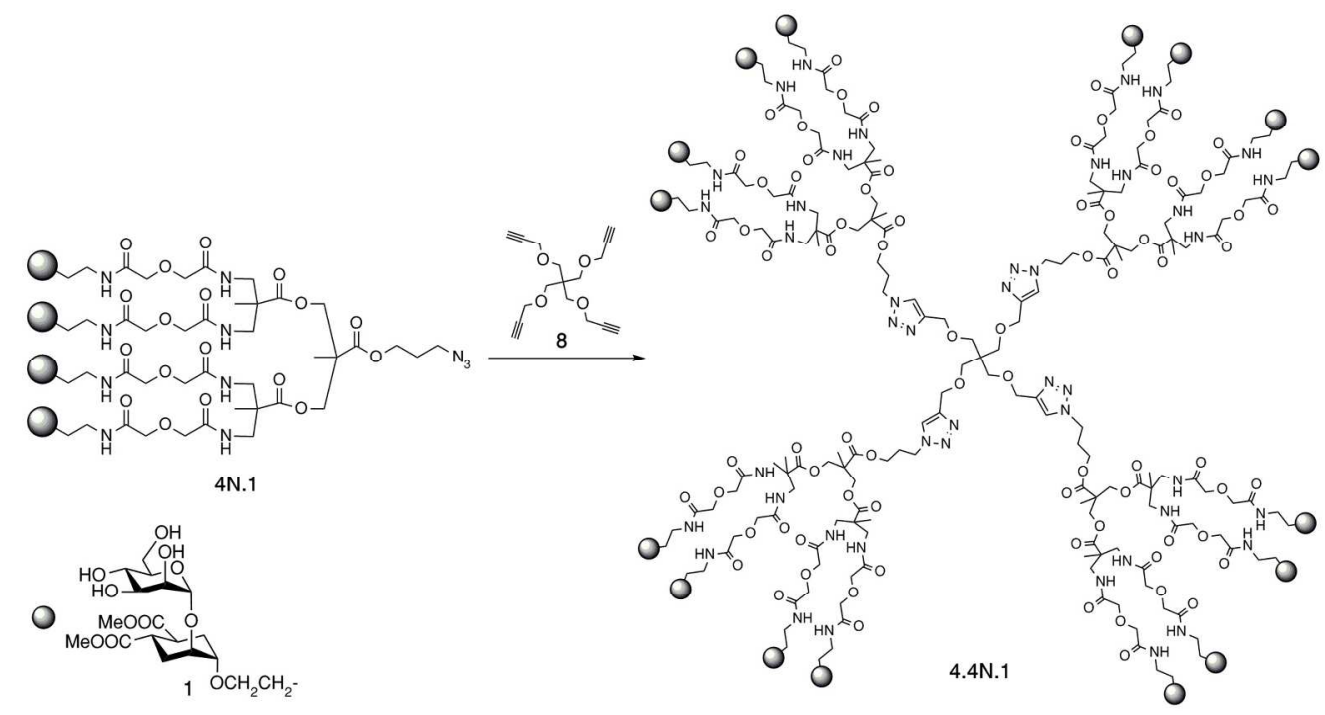

Scheme 2: Synthesis of the 16-valent compound 4.4N.1 from dendron 4N.1.

$180 \times 97 \mathrm{~mm}(300 \times 300 \mathrm{DPI})$ 